\title{
Long-range action of Nodal requires interaction with GDF1
}

\author{
Chinatsu Tanaka, ${ }^{1}$ Rui Sakuma, ${ }^{1,3}$ Tetsuya Nakamura, ${ }^{1}$ Hiroshi Hamada, ${ }^{1,4}$ and Yukio Saijoh ${ }^{1,2}$ \\ ${ }^{1}$ Developmental Genetics Group, Graduate School of Frontier Biosciences, Osaka University, and CREST, Japan Science and \\ Technology Corporation (JST), Suita, Osaka 565-0871, Japan; ${ }^{2}$ Department of Neurobiology and Anatomy, and The Eccles \\ Program in Human Molecular Biology and Genetics, University of Utah, Salt Lake City, Utah 84112, USA
}

GDF1 (growth/differentiation factor 1), a Vg1-related member of the transforming growth factor- $\beta$ superfamily, is required for left-right patterning in the mouse, but the precise function of GDF1 has remained largely unknown. In contrast to previous observations, we now show that GDF1 itself is not an effective ligand but rather functions as a coligand for Nodal. GDF1 directly interacts with Nodal and thereby greatly increases its specific activity. Gdf1 expression in the node was found necessary and sufficient for initiation of asymmetric Nodal expression in the lateral plate of mouse embryos. Coexpression of GDF1 with Nodal in frog embryos increased the range of the Nodal signal. Introduction of Nodal alone into the lateral plate of Gdf1 knockout mouse embryos did not induce Lefty 1 expression at the midline, whereas introduction of both Nodal and GDF1 did, showing that GDF1 is required for long-range Nodal signaling from the lateral plate to the midline. These results suggest that GDF1 regulates the activity and signaling range of Nodal through direct interaction.

[Keywords: Embryonic patterning; GDF1; left-right axis; Nodal; signaling]

Supplemental material is available at http://www.genesdev.org.

Received May 31, 2007; revised version accepted October 29, 2007.

Despite recent progress in understanding of how leftright (L-R) asymmetry is generated during vertebrate development (Capdevila et al. 2000; Hamada et al. 2002), knowledge of this process remains limited, with many important questions still unanswered. One such question concerns the mechanism by which the signal responsible for the generation of L-R asymmetry is transferred from the node to the lateral plate. This signal, whose identity remains unknown, is generated in the node, and its arrival in the left lateral plate induces the asymmetric expression of Nodal. Although the L-R symmetry-breaking event in the mouse embryo is the leftward flow of extraembryonic fluid in the node (Nonaka et al. 1998), it is not known how this so-called nodal flow achieves its effect. It may thus transport an unknown determinant toward the left side of the node cavity, or it may generate mechanical stress that is recognized by mechanosensors.

Signaling molecules expressed in the node are essential for correct L-R patterning of the lateral plate, and they may play a role in transfer of the L-R asymmetric signal. In particular, Nodal is expressed bilaterally in the node (in perinodal crown cells) before the onset of its

\footnotetext{
${ }^{3}$ Present address: The Hospital for Sick Children, 555 University Avenue, Toronto, Ontario M5G 1X8, Canada.

${ }^{4}$ Corresponding author.

E-MAIL hamada@fbs.osaka-u.ac.jp; FAX 81-6-6878-9846.

Article is online at http://www.genesdev.org/cgi/doi/10.1101/gad.1623907.
}

expression in the left lateral plate mesoderm (LPM). Genetic evidence (Brennan et al. 2002; Saijoh et al. 2003) has shown that Nodal expression in the node is essential for subsequent Nodal expression in the left LPM. The specific elimination of Nodal expression in the perinodal region thus prevents Nodal expression in the left LPM (Brennan et al. 2002; Saijoh et al. 2003). The Nodal antagonist Dante (also known as Cerl2) is also expressed in the perinodal region before Nodal expression begins in the left LPM (Pearce et al. 1999). Cerl2 is expressed in an L-R asymmetric manner, with its expression on the right side being substantially higher than that on the left side. Mice that lack Cerl2 show bilateral or right-sided expression of Nodal in the LPM (Marques et al. 2004), suggesting that this Nodal antagonist produced in the node regulates the asymmetric expression of Nodal in the LPM. Nodal may thus play a role in signal transfer from the node to the left LPM, or the Nodal itself may travel from the node to the left LPM.

Like Nodal, growth/differentiation factor 1 (GDF1), a member of the transforming growth factor- $\beta$ (TGF- $\beta$ ) superfamily of proteins that is most closely related to Xenopus $\mathrm{Vg1}$, is expressed bilaterally in the perinodal region of mouse embryos. Mice that lack GDF1 do not manifest asymmetric expression of Nodal in the LPM, and exhibit right isomerism of visceral organs (Rankin et al. 2000). Similarities in the expression domains and mutant phenotypes of Gdf1 and Nodal suggest that GDF1 may play a role in signal transfer from the node to the 
LPM by interacting with Nodal. However, the precise role of GDF1 in L-R patterning has remained unknown. Gdf1 is expressed not only in the perinodal region but also in the LPM at the early somite stage, suggesting that the lack of Nodal expression in the LPM of Gdf1-null mice may be due to the absence of GDF1 in the LPM. Furthermore, GDF1 signaling is mediated by components of the Nodal signaling pathway, and overexpression of GDF1 in frog embryos, or cultured cells induces activation of a Nodal-responsive reporter gene (Wall et al. 2000), suggesting that GDF1 may contribute to L-R patterning independently of Nodal.

Genetic evidence suggests that Gdf1 and Nodal are required for transfer of the $\mathrm{L}-\mathrm{R}$ asymmetric signal from the node to the lateral plate, although their precise roles remain unknown. To provide insight into the mechanism by which the asymmetric signal is transferred from the node to the LPM, we examined the role of GDF1 in L-R patterning. Our data suggest that GDF1 itself is not an active ligand, but that it is required in the node as a partner of Nodal for L-R patterning of the LPM. Formation of a heterodimer with GDF1 results in a marked increase in Nodal activity, and is required for long-range action of Nodal, such as that which contributes to signal transfer between the node and the LPM.

\section{Results}

Gdf1 expression in the node is necessary and sufficient for initiation of asymmetric Nodal expression in the $L P M$

Gdf1-null mice manifest right isomerism, with most mutant embryos lacking asymmetric Nodal expression in the LPM (Rankin et al. 2000). However, our re-examination of the L-R defects of $G d f 1^{-/-}$mice revealed that all mutant embryos examined lacked expression of Nodal (20 of 20) and Pitx2 (23 of 23) in the left LPM (Supplementary Fig. S1A-D), suggesting that GDF1 is absolutely required for left-sided gene expression in the LPM. Nodal expression in the node was maintained in all mutant embryos (Supplementary Fig. S1A,B), consistent with previous observations (Rankin et al. 2000). At the early somite stage, Gdf1 is expressed in several domains including the node and the LPM, with expression in the node being confined to perinodal crown cells, which also express Nodal (Supplementary Fig. S1E,F). Two-color in situ hybridization confirmed that Gdf1 and Nodal are coexpressed in perinodal crown cells and in the left LPM cells (Supplementary Fig. S1G). The phenotype of $G d f 1^{-/-}$mice is thus similar to that of mice that lack Nodal expression in the node (Brennan et al. 2002).

Given that Gdf1 is expressed both in the node and in the LPM, it was possible that the lack of Nodal expression in the LPM of $G d f 1^{-/-}$embryos was due to the absence of GDF1 in the node, in the LPM, or in both regions. To distinguish among these possibilities, we constructed transgenes that would confer expression of $G d f 1$ specifically in the node or in the LPM, and examined whether these transgenes were able to rescue the L-R defects of $G d f 1^{-/-}$mice.

For a transgene that would confer expression of $G d f 1$ in the node (node- $T g$ ) (Fig. 1A), the Gdf1 cDNA linked to IRES-lacZ (an internal ribosome entry site linked to lacZ) was placed under the control of the node-specific enhancer (NDE) of Nodal (Krebs et al. 2003). For a transgene that would confer bilateral expression of $G d f 1$ in the LPM, the Gdf1 cDNA linked to IRES-lacZ was positioned under the control of the $11-\mathrm{kb}$ upstream region of Cryptic (LPM-Tg) (Fig. 1A). Permanent mouse lines expressing each Gdf1-IRES-lacZ cassette with the desired specificity were established (Fig. 1A).

Expression of $L P M-T g$ alone failed to restore Nodal

A
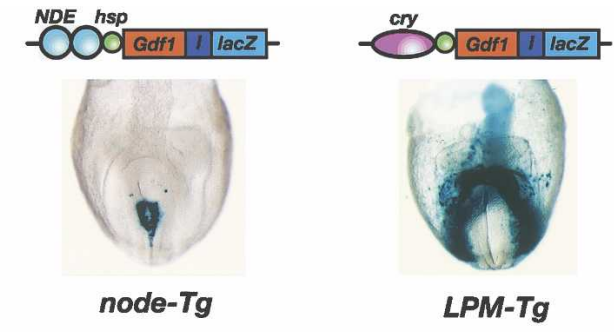

Gdf1 -/-

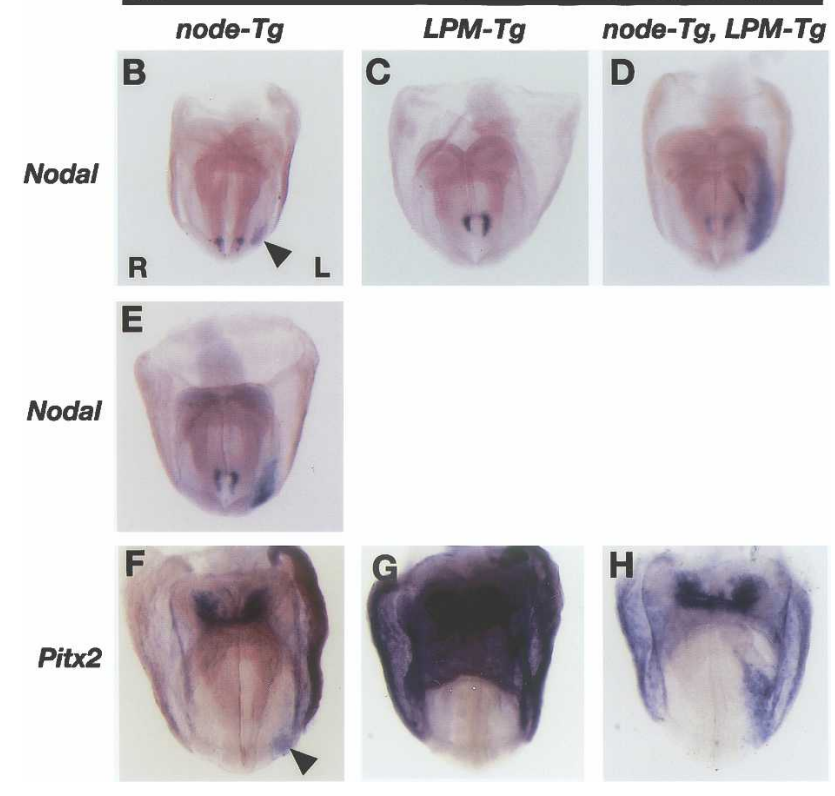

Figure 1. Restoration of asymmetric Nodal expression in the LPM of $G d f 1^{-/-}$embryos by expression of Gdf1 transgenes. (A) Schematic representations of two Gdf1 transgenes (node- $T g$ and $L P M-T g$ ) are shown above corresponding transgenic embryos at the early somite stage stained with the $\beta$-galactosidase substrate X-gal. (hsp) hsp68 promoter; (I) IRES; (cry) 11-kb upstream region of Cryptic. $(B-H)$ Whole-mount in situ hybridization analysis of the expression of Nodal $(B-E)$ and Pitx2 $(F-H)$ in $G d f 1^{-1}$ embryos harboring the indicated transgenes at the early somite stage. In some embryos harboring node- $\mathrm{Tg}$, expression of Nodal was confined to the distal side of the left LPM and did not fully extend along the A-P axis $(B$, arrowhead), whereas in others it did expand along this axis $(E)$. $L P M-T g$ failed to restore expression of Nodal or Pitx2 in the left LPM. The presence of both transgenes fully restored Nodal and Pitx2 expression in the left LPM. 
expression in the left LPM of all (four of four) $G d f 1^{-/-}$ embryos examined (Fig. 1C). Expression of Pitx2 was also absent in all (eight of eight) $G d f 1^{-/}$; LPM-Tg embryos (Fig. 1G). In contrast, expression of node-Tg in Gdf1-/embryos resulted in a partial restoration of Nodal expression in the left LPM. In most (four out of six) of the embryos examined, Nodal expression was confined to a small region of the LPM adjacent to the node and did not expand along the anteroposterior (A-P) axis (Fig. 1B). In the remaining (two out of six) embryos, however, the Nodal expression domain in the LPM did expand along the A-P axis (Fig. 1E). Consistent with this partial rescue of Nodal expression, L-R defects of abdominal organs were normalized in $G d f 1^{-1-}$; node- $T g$ newborn mice (Table 1; Supplementary Fig. S2). Thoracic organs, however, including the heart and lungs, remained abnormal (Table 1; Supplementary Fig. S2), and most $G d f 1^{-1-}$; node- $T g$ mice died within several days after birth as a result of cardiac abnormalities. Expression of both LPM$\mathrm{Tg}$ and node- $\mathrm{Tg}$ resulted in complete restoration of asymmetric expression of Nodal and Pitx2 in the LPM (Fig. 1D,H). Indeed, $G d f 1^{-/-}$mice harboring both node- $T g$ and $L P M-T g$ developed normally to term with no apparent defect in cardiac looping (data not shown) and were fertile, suggesting that the developmental defects of the knockout mice were completely rescued by expression of both transgenes. These results thus suggested that GDF1 in the node is essential and sufficient for initiation of asymmetric Nodal expression in the LPM. GDF1 in the LPM is also required for expansion of Nodal expression in the LPM along the A-P axis.

GDF1 is not an effective ligand alone but enhances Nodal activity

Coexpression of Gdf1 and Nodal in perinodal crown cells (Supplementary Fig. S1G), the phenotypic similarity between $G d f 1^{-/-}$mice and mice lacking Nodal specifically in the node (Brennan et al. 2002), and the abso- lute requirement of GDF1 in the node for initiation of Nodal expression in the left LPM suggested that GDF1 might be necessary for the proper function of Nodal produced in the node. Previous studies have suggested that GDF1 signaling is mediated by components of the Nodal signaling pathway (Wall et al. 2000; Cheng et al. 2003). In these studies, a chimeric construct comprising the mature region of GDF1 fused to the proregion of bone morphogenetic protein (BMP) or Activin was active in animal cap assays, whereas native GDF1 was either inactive or not tested. It was anticipated that fusion to the proregion of a different member of the TGF- $\beta$ superfamily would facilitate release of mature GDF1 from the precursor protein.

We tested the activity of GDF1 in the Xenopus animal cap assay with a Nodal-responsive luciferase reporter gene, (n2) ${ }_{7}$ luc (Saijoh et al. 2000; Sakuma et al. 2002). Injection of mRNA encoding a BMP-GDF1 fusion protein resulted in activation of the reporter in animal caps (Supplementary Fig. S3A). However, injection of even a large amount (1000 pg) of mRNA encoding native GDF1 failed to activate the reporter (Fig. 2A). The mature form of GDF1 was produced by animal caps as well as by oocytes injected with mRNA for GDF1 (Supplementary Fig. S3C,D), suggesting that the native GDF1 precursor protein is cleaved in this system, albeit with a lower efficiency than is the BMP-GDF1 fusion protein. Unexpectedly, however, the native GDF1 protein greatly increased the activity of Nodal (Fig. 2B,C). This facilitative effect of GDF1 was not apparent in animal caps expressing either of the Nodal antagonists Lefty1 or Lefty2 (Fig. 2C). Phosphorylation of Smad2 induced by Nodal was also greatly enhanced by GDF1 (Fig. 2D). These results suggested that GDF1 is not an active ligand by itself but that it enhances Nodal activity mediated by the canonical Nodal signaling pathway.

We also examined whether GDF1 homologs of other vertebrates exhibit similar activity. Among members of the TGF- $\beta$ superfamily in zebrafish, a protein encoded by $z D V R-1$ (now regarded as the zebrafish ortholog of

Table 1. $L-R$ defects of $\mathrm{Gdf}^{-/-}$mice and their partial rescue by expression of node- $\mathrm{Tg}$

\begin{tabular}{|c|c|c|c|c|c|}
\hline \multirow[b]{2}{*}{ Organ } & \multirow[b]{2}{*}{ Position } & \multicolumn{3}{|c|}{$G d f 1^{-/-}$} & \multirow[b]{2}{*}{$G d f 1^{-1-} ;$ node $-\mathrm{Tg}$} \\
\hline & & I & II & III & \\
\hline Heart malformation & & + & + & + & + \\
\hline Right pulmonary isomerism & & + & + & + & + \\
\hline Stomach and spleen & Normal & + & & & + \\
\hline & Reversed & & + & + & \\
\hline Liver & $\begin{array}{l}\text { Normal } \\
\text { Reversed } \\
\text { Symmetric }\end{array}$ & + & + & + & + \\
\hline Kidneys & $\begin{array}{l}\text { Normal } \\
\text { Reversed }\end{array}$ & + & + & + & + \\
\hline Relative positions of vena cava and aorta & $\begin{array}{l}\text { Normal } \\
\text { Reversed }\end{array}$ & + & + & + & + \\
\hline Number of mice examined & & 1 & 4 & 2 & 5 \\
\hline
\end{tabular}

Various visceral organs of $G d f 1^{-/-}$and $G d f 1^{-/-}$; node-Tg newborn mice were examined for their position and morphology. Three patterns (I, II, and III) of defects were observed in $G d f 1^{-1-}$ mice. The L-R defects of abdominal organs such as stomach, spleen, liver, and kidneys were rescued in $\mathrm{Gdf1}^{-/-}$; node- $\mathrm{Tg}$ mice. 

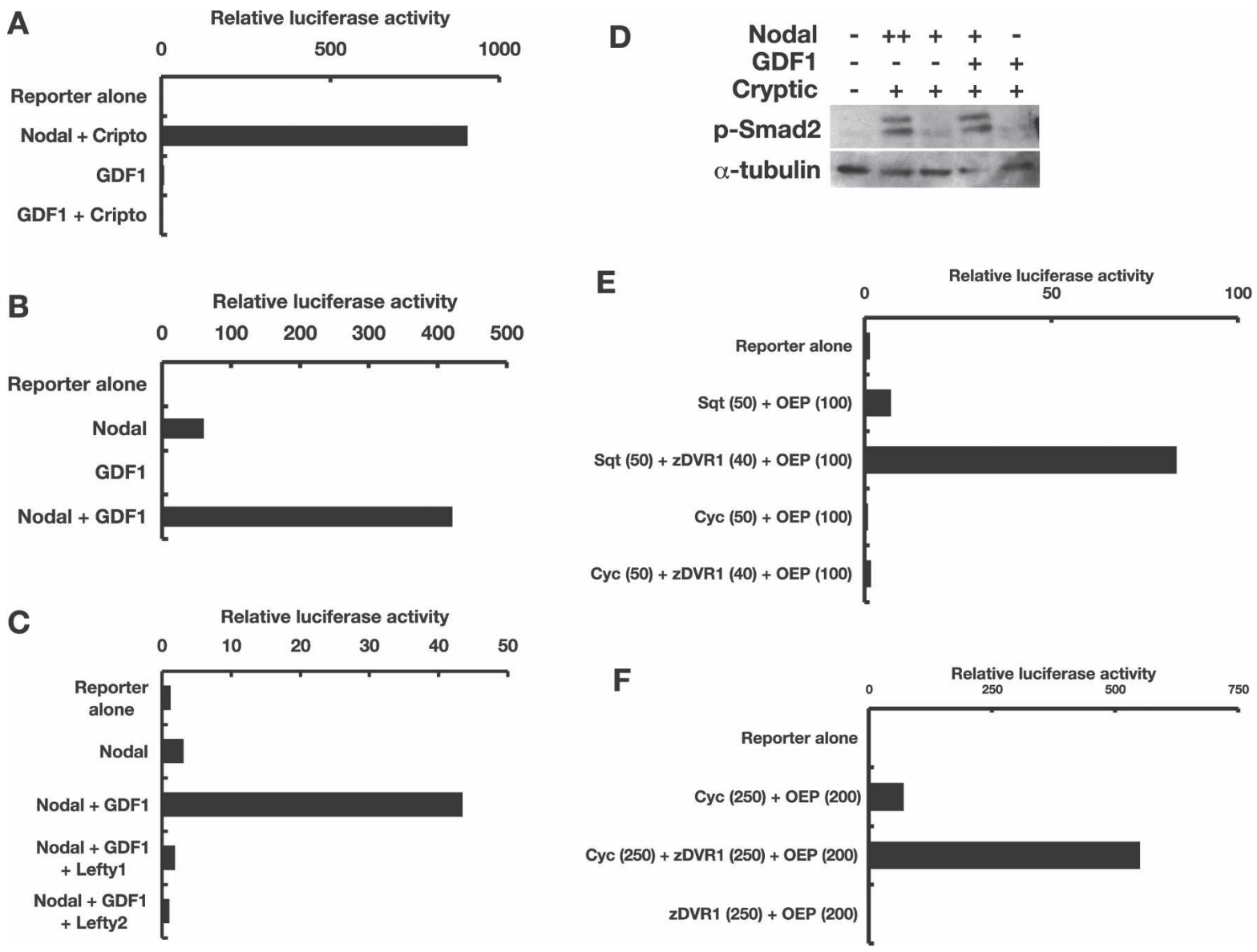

Figure 2. GDF1 is not an active ligand but enhances Nodal activity. $(A-C)$ The activity of the Nodal-responsive reporter (n2) 7 luc in the Xenopus animal cap assay was determined after injection of mRNAs for Nodal (10 pg), GDF1 (1000 pg), or the Nodal coreceptor Cripto (20 pg) (A); of mRNAs for Nodal (2 pg) or GDF1 (40 pg) (B); or of mRNAs for Nodal (2 pg), GDF1 (40 pg), Lefty1 (50 pg), or Lefty2 $(50 \mathrm{pg})(C)$. All embryos in $B$ and $C$ were also injected with $100 \mathrm{pg}$ of the mRNA for the Nodal coreceptor Cryptic. (D) Xenopus embryos were injected with mRNAs for Nodal $\left(++, 50 \mathrm{pg}_{+}+, 10 \mathrm{pg}\right)$, GDF1 (40 pg), or Cryptic (100 pg), as indicated, after which animal caps were subjected to immunoblot analysis with antibodies to phospho-Smad2 (p-Smad2) or to $\alpha$-tubulin (loading control). (E, $F$ ) The animal cap assay was also performed with mRNAs for zDVR1, Squint (Sqt), Cyclops (Cyc), or Flag-tagged OEP (OEP), as indicated. Injected mRNA amounts are shown in picograms (in parentheses).

Xenopus Vg1) shows the highest similarity and may be equivalent to mouse GDF1 (Dohrmann et al. 1996). Injection of mRNA encoding the native zDVR1 protein (250 pg) in our animal cap assay did not activate expression of the reporter gene (data not shown); a similar result was obtained when the mRNA for zDVR1 was injected together with Oep mRNA, which encodes an EGF-CFC protein (Fig. 2F). However, coinjection of zDVR1 mRNA with zebrafish Squint or Cyclops mRNA resulted in a marked increase in the activity of Squint or Cyclops (Fig. 2E,F). These results suggested that the function of GDF1 is conserved in zebrafish, given that zDVR1 was inactive by itself but enhanced the activities of Nodal-related factors.

\section{Heterodimerization with GDF1 increases the specific activity of Nodal}

The ability of GDF1 to enhance Nodal signaling, coexpression of Gdf1 and Nodal in the node (Supplementary
Fig. S1G), and the phenotypic similarity between $G d f 1^{-/-}$ mice (Rankin et al. 2000) and Nodal mutant mice (Brennan et al. 2002) suggested that the TGF- $\beta$-related factors encoded by these two genes might interact with each other. To determine whether Nodal and GDF1 indeed interact to form a heterodimer, we prepared conditioned medium from frog oocytes that had been injected with mRNAs encoding GDF1 and Flag epitope-tagged Nodal or with mRNAs for Nodal and Flag-GDF1. Addition of the Flag tag did not affect the activity of Nodal or GDF1 in the animal cap assay (data not shown). The conditioned media were then subjected to immunoprecipitation with antibodies to Flag, and the resulting immunoprecipitates were analyzed with an immunoblot assay. The amount of mature Nodal in conditioned medium was not affected by coexpression of Flag-GDF1 (Fig. 3B), suggesting that GDF1 does not affect the stability of Nodal, the proteolytic cleavage of the Nodal precursor, or the secretion of Nodal into the culture medium. Analysis of immunoprecipitates under reducing condi- 
A

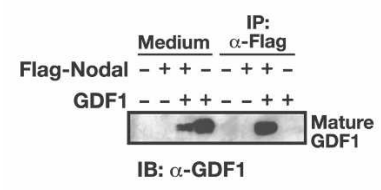

C

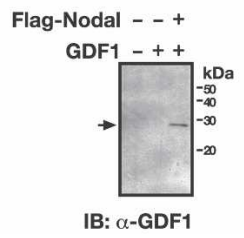

B

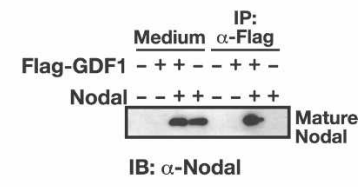

D

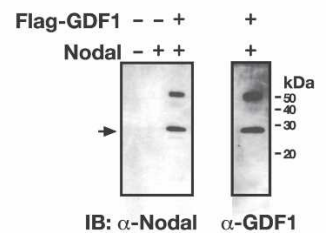

Figure 3. GDF1 Interacts with Nodal. $(A)$ Conditioned medium prepared from Xenopus oocytes expressing Flag-Nodal or GDF1, as indicated, was subjected to immunoprecipitation (IP) with antibodies to Flag ( $\alpha$-Flag). The resulting precipitates as well as the conditioned medium were subjected to immunoblot analysis (IB) under reducing conditions with antibodies to GDF1 $(\alpha$-GDF1). (B) Conditioned medium prepared from Xenopus oocytes expressing Flag-GDF1 or Nodal, as indicated, was subjected to immunoprecipitation with antibodies to Flag. The resulting precipitates as well as the conditioned medium were subjected to immunoblot analysis under reducing conditions with antibodies to Nodal. $(C)$ Conditioned medium prepared from Xenopus oocytes expressing Flag-Nodal or GDF1, as indicated, was subjected to immunoprecipitation with antibodies to Flag, and the resulting precipitates were subjected to immunoblot analysis under nonreducing conditions with antibodies to GDF1. Arrow indicates a Flag-Nodal-GDF1 heterodimer. $(D)$ Conditioned medium prepared from Xenopus oocytes expressing Flag-GDF1 or Nodal, as indicated, was subjected to immunoprecipitation with antibodies to Flag, and the resulting precipitates were subjected to immunoblot analysis under nonreducing conditions with antibodies to Nodal or to GDF1. Arrow indicates a Flag-GDF1-Nodal heterodimer. The lower-mobility band corresponds to a heterodimer of Nodal and of Flag-GDF1 that was not cleaved at the proteolytic cleavage site.

tions revealed that GDF1 was associated with FlagNodal (Fig. 3A), and that Nodal was associated with FlagGDF1 (Fig. 3B). Analysis of the immunoprecipitates under nonreducing conditions revealed that the FlagNodal-GDF1 and Flag-GDF1-Nodal complexes migrated at a position corresponding to a molecular size of $\sim 27$ kDa (Fig. 3C,D), indicating that Nodal and GDF1 interact to form a heterodimer. Hemagglutinin epitope (HA)tagged Nodal interacted with Flag-Nodal and Flag-GDF1 with similar efficiencies, suggesting that both the Nodal homodimer and the Nodal-GDF1 heterodimer exist when both proteins are coexpressed at similar levels (Supplementary Fig. S4).

To examine whether its interaction with GDF1 affects the specific activity of Nodal, we compared the activity of the GDF1-Nodal heterodimer with that of the Nodal homodimer. Conditioned medium containing Nodal alone, GDF1 alone, or Nodal plus GDF1 was prepared with frog oocytes, and the abundance of each protein was determined by immunoblot analysis. Each conditioned

medium was then examined for its ability to activate the Nodal-responsive reporter (n2) 7 luc. Assay of equivalent levels of Nodal protein revealed that the activity of the Nodal-GDF1 heterodimer was 100 times that of Nodal alone (Fig. 4A,B). GDF1 alone was inactive in the assay. The Nodal-GDF1 heterodimer was inactive in the absence of Cryptic (Supplementary Fig. S3F), indicating that the heterodimer acts through Cryptic. When GDF1conditioned medium and Nodal-conditioned medium were prepared separately and mixed, the resulting mixture failed to activate the reporter gene (Fig. 4C), suggesting that the functional Nodal-GDF1 heterodimer is formed intracellularly. Furthermore, the medium conditioned with GDF1 did not efficiently stimulate reporter gene expression in animal caps injected with Nodal mRNA (Fig. 4D), suggesting that it is unlikely that GDF1 induces an unknown factor that synergizes with the Nodal pathway. Together, these results suggest that interaction with GDF1 increases the specific activity of Nodal by two orders of magnitude.

A form of GDF1 (cmGDF1) in which an amino acid residue required for proteolytic cleavage of the proprotein is mutated failed to yield mature GDF1 yet was still able to interact with Nodal (Supplementary Fig. S5A,G). The cmGDF1 mutant was not only unable to enhance Nodal activity but actually inhibited Nodal activity (Supplementary Fig. S5C), suggesting that interaction with mature GDF1 is required for enhancement of Nodal activity. Most members of the TGF- $\beta$ superfamily are thought to form homo- and heterodimers through cysteine residues. We therefore mutated cysteine residues of GDF1 and Nodal to generate the mutants dmGDF1 and dmNodal, respectively (Supplementary Fig. S5A). The dmNodal mutant was as active as the wild-type Nodal and was able to interact with wild-type GDF1 (Supplementary Fig. S5B,D), whereas dmGDF1 maintained the ability to interact with Nodal and to enhance Nodal activity (Supplementary Fig. S5B,E). However, dmGDF1 failed to enhance the activity of dmNodal, even though it interacted with dmNodal in the immunoprecipitation assay (Supplementary Fig. S5B,F). Thus, dmGDF1 and dmNodal are able to interact physically with each other, but not in a manner that results in the stimulation of Nodal activity, suggesting that mutation of the cysteine residues affects a higher-order interaction of the two proteins.

\section{Long-range action of Nodal requires GDF1 in frogs and mice}

Both Nodal and GDF1 produced in the node are essential for asymmetric Nodal expression in the LPM. Evidence also indicates that Nodal produced in the node travels to the LPM, where it activates asymmetric Nodal expression (Brennan et al. 2002; Saijoh et al. 2005). These observations suggest that GDF1 may be required for longrange action of Nodal.

We investigated this possibility first with a reporter assay in frog embryos. A reporter mixture, consisting of the Nodal-responsive lacZ reporter gene (f1) ${ }_{6}$ lacZ (Saijoh 
A
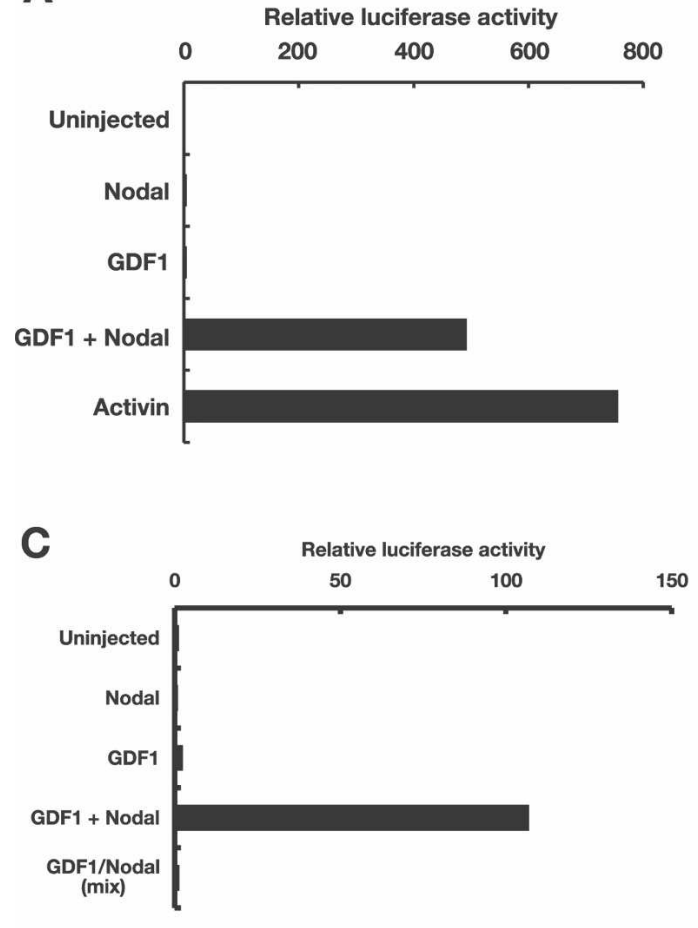

B

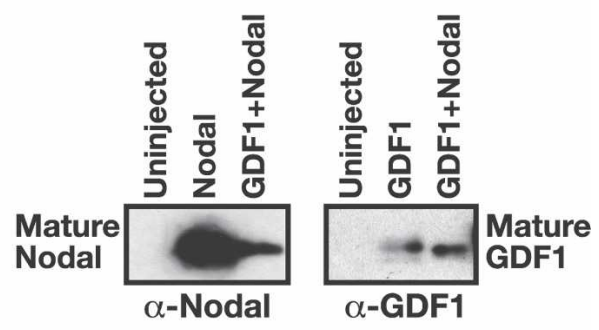

D

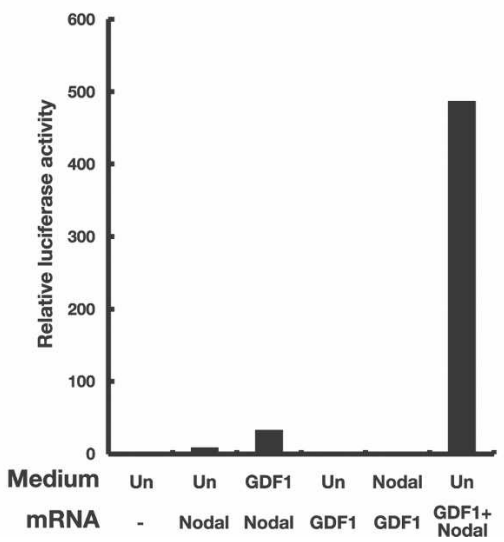

Figure 4. Interaction with GDF1 increases Nodal activity. (A) Conditioned medium prepared from Xenopus oocytes expressing Nodal, GDF1, or Activin, as indicated, was assayed for activity in a Xenopus animal cap assay with the Nodal-responsive reporter (n2) 7 luc. (B) Immunoblot analysis of the conditioned media $(10 \mu \mathrm{L})$ used for the assay in $A$. The GDF1 protein coexpressed with Nodal in frog oocytes migrated slightly faster than did that expressed in the absence of Nodal. This was also true when GDF1 was expressed with or without Nodal in COS cells (Supplementary Fig. S6). (C) Conditioned medium prepared from Xenopus oocytes expressing Nodal, GDF1, or both proteins (GDF1 + Nodal) was assayed for activity as in A. For "GDF1/Nodal (mix)," conditioned medium for GDF1 and that for Nodal were prepared separately and mixed. (D) Frog embryos were injected with (n2) 7 luc and mRNAs for Nodal (2 pg) or GDF1 (40 pg) as indicated (mRNA). Animal caps prepared from the embryos were then cultured in conditioned medium prepared from Xenopus oocytes expressing Nodal or GDF1 as indicated (Medium), after which the activity of (n2) 7 luc was determined. (Un) Uninjected. All embryos in $A, C$, and $D$ were also injected with $100 \mathrm{pg}$ of the mRNA for Cryptic.

et al. 2000) and Cryptic mRNA, and an effector mixture, comprising Nodal mRNA with or without Gdf1 mRNA, were injected separately into two blastomeres of frog embryos at the 32- or 64-cell stage (Fig. 5A). Texas Red lysine dextran (TRLDx) and fluorescein lysine dextran (FLDx) were included in the reporter and effector mixtures, respectively, to allow monitoring of the fates of the injected cells. Animal caps were prepared at stage 8.5 , incubated for $3 \mathrm{~h}$, and stained with X-gal. When the two mixtures were injected into neighboring blastomeres, the reporter gene was activated regardless of the absence or presence of $G d f 1$ mRNA (Fig. 5B,D,F). In contrast, when the two mixtures were injected into blastomeres that were separated by one or two cells, reporter activation was dependent on the presence of Gdf1 mRNA (Fig. 5E,G,H). Examination of TRLDx and FLDx fluorescence confirmed that the two groups of cells descended from the injected cells remained separated at the end of the assay (Fig. 5C). These results suggested that Nodal is able to function over a long distance only in the presence of GDF1.

We then examined whether GDF1 is required for long- range action of Nodal in mouse embryos. One event that requires long-range action of Nodal is the induction of Lefty1 expression at the midline during L-R patterning. Expression of Lefty1 in the floor plate is thus induced directly by Nodal protein that is produced in the left LPM (Yamamoto et al. 2003). Nodal synthesized in the LPM must therefore travel to the midline to achieve this effect. Gdf1-/- embryos lack Lefty1 expression because Nodal expression is absent in the LPM (data not shown). We therefore introduced a Nodal expression vector with or without a $G d f 1$ expression vector into the right LPM of $G d f 1^{+/-}$or $G d f 1^{-/-}$embryos by lipofection, and determined whether expression of Lefty1 was induced at the midline (Fig. 6A). An expression vector for green fluorescent protein (GFP) was also included in the lipofection mixture to verify the site of injection (Fig. 6C,E). Introduction of the Nodal vector alone or together with the Gdf1 vector into the right LPM of $G d f 1^{+/-}$embryos induced Nodal expression in the right LPM and Lefty1 expression in the right floor plate, as expected (data not shown). Introduction of the Nodal vector alone did not induce Lefty1 expression in any of the five $G d f 1^{-/-}$em- 
A
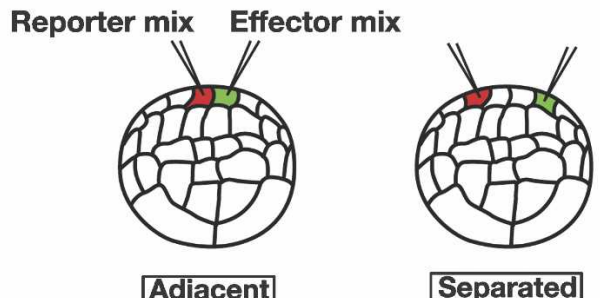

B

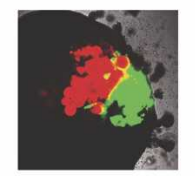

Adjacent, Nodal

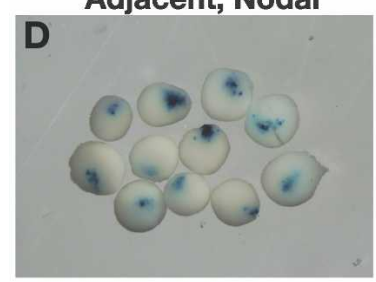

Adjacent, Nodal + GDF1

$\mathbf{F}$

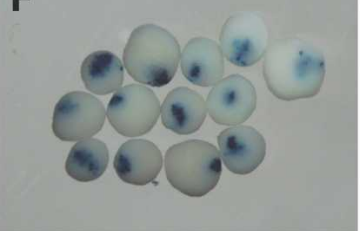

Reporter mix alone

H
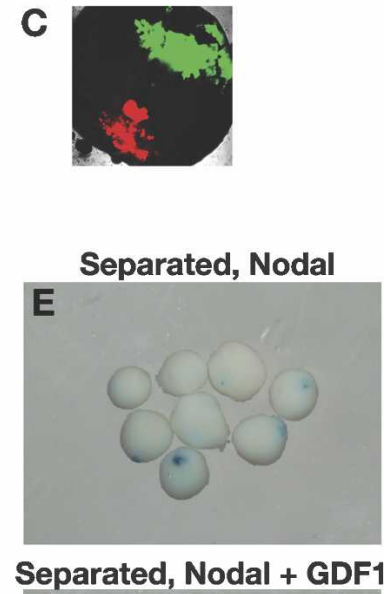

G

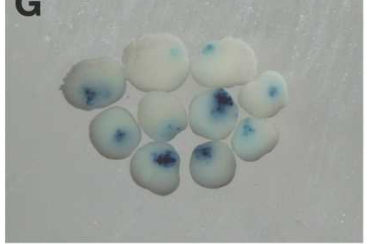

Gdf1 $1^{-/}$embryos tested (Fig. 6D). Examination of transverse sections showed that Lefty1 expression was induced in the floor plate on the right side (Fig. 6F), confirming that the expression domain was attributable to the Nodal and Gdf1 expression vectors. These results indicated that GDF1 is required for long-range action of Nodal (from the LPM to the midline) in the mouse embryo.

A
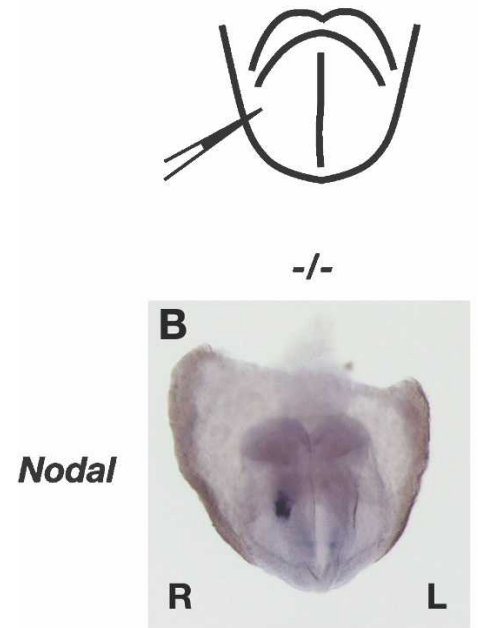

C

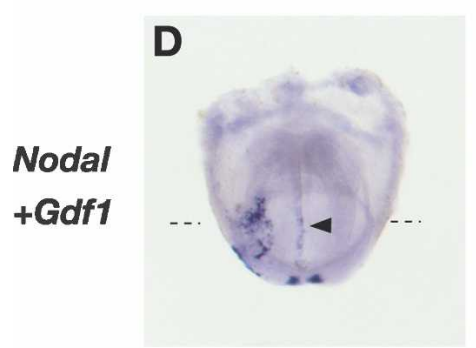

$\mathbf{L}$

E

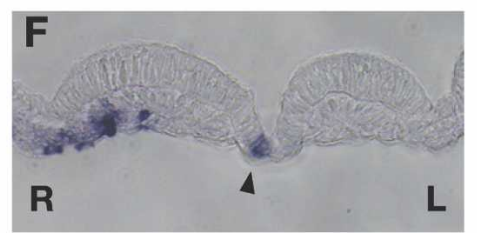

Figure 5. GDF1 increases the range of the Nodal signal in frog embryos. $(A-C)$ Experimental strategy. The Nodal-responsive reporter (f1) ${ }_{6} l a c Z$, mRNAs for Cryptic (125 pg) and the Activin type I receptor ALK4 (50 pg), and TRLDx were injected into a single blastomere of a 32- or 64-cell stage Xenopus embryo. (A) Nodal mRNA (250 pg), with or without Gdf1 RNA (225 pg), was injected together with FLDx into either an adjacent blastomere or a blastomere separated by one or two cells. Animal caps were prepared at stage 8.5 , cultured for $3 \mathrm{~h}$, and stained with X-gal. The fluorescence of TRLDx and FLDx was also monitored to reveal descendents of the adjacent $(B)$ or separated $(C)$ injected cells. $(D-H)$ X-Gal-stained animal caps for adjacent $(D, F)$ or separated $(E, G)$ blastomeres injected with Nodal mRNA alone $(D, E)$ or with Nodal mRNA plus $G d f 1$ mRNA $(F, G)$ in the effector mix. $(H)$ An X-gal-stained animal cap derived from an embryo injected with only the reporter mix is also shown.

bryos tested (Fig. 6B). However, introduction of both Nodal and Gdf1 vectors into the right LPM induced Lefty1 expression at the midline of eight of the nine 


\section{Discussion}

GDF1 is not an effective ligand alone but functions as a coligand of Nodal

Previous studies have suggested that GDF1 alone activates intracellular signaling when overexpressed (Wall et al. 2000; Cheng et al. 2003). However, these previous studies were performed with an expression vector or mRNA for a fusion protein containing the mature region of GDF1 and the proregion of BMP or Activin; mRNA for native GDF1 was inactive (Wall et al. 2000). We obtained similar results in the present study. Furthermore, signaling by GDF1 was suggested to be mediated by the Nodal signaling pathway (Cheng et al. 2003). Similar observations have been made for frog $\mathrm{Vgl}$, which shows sequence similarity to GDF1. Thus, injection of mRNA for native Vg1 into frog embryos did not induce mesoderm formation, whereas injection of an mRNA for a fusion protein containing the proregion of BMP and the mature region of Vg1 did so efficiently (Thomsen and Melton 1993; Kessler and Melton 1995).

The interpretation of these previous observations was that mRNA encoding native GDF1 was not active because of a low efficiency of the proteolytic cleavage responsible for conversion of the preproprotein into the mature protein. Several lines of evidence obtained in the present study, however, suggest that GDF1 is not an effective ligand by itself. First, mRNA for native GDF1 generated the mature form of GDF1 in Xenopus animal caps and oocytes, albeit with a reduced efficiency compared with mRNA for a BMP-GDF1 fusion protein. Despite its ability to produce the mature GDF1 protein, injection of animal caps with even large amounts (1 ng) of the mRNA for native GDF1 did not result in the activation of a Nodal-responsive reporter gene. Second, conditioned medium containing GDF1 alone was inactive in this assay, whereas that containing both GDF1 and Nodal was highly active. Third, restoration of Gdf1 expression in the lateral plate of $G d f 1^{-/-}$mouse embryos with an LPM-specific transgene was unable to restore asymmetric expression of Nodal in the LPM.

Although there is no apparent discrepancy between the previous observations and our present results, our data suggest that, under physiological conditions, GDF1 is not an effective ligand but functions as a coligand of Nodal. It is unclear how interaction with GDF1 enhances Nodal activity, but it may increase the affinity of Nodal for its receptor.

\section{GDF1 is required for long-range action of Nodal}

Our data suggest that GDF1 is required for long-range action of Nodal in the mouse embryo. This may also be the case in the zebrafish embryo, given that zDVR1 enhanced the activity of Squint and of Cyclops. Short-range action of Nodal may not require GDF1, given that Nodal expression in the LPM was rescued, at least partially, in Gdf1 $1^{-1}$; node- $\mathrm{Tg}$ embryos.

Long-range action of Nodal is likely required for at least two events during L-R patterning (Fig. 7A). First, expression of Lefty 1 at the midline is directly induced by Nodal produced in the left LPM (Yamamoto et al. 2003). Given that the cells located between the midline and the the LPM do not express Cryptic (Shen et al. 1997) or Cripto (Dono et al. 1993) and thus would not be expected to be responsive to the Nodal signal, Nodal produced in the left LPM must travel to the midline in order to induce Lefty1 expression. Our results suggest that Nodal travels this long distance as a heterodimer with GDF1. Second, Nodal may similarly travel the long distance from the node to the lateral plate. Our transgenic rescue experiments showed that expression of Gdf1 in the node is required for asymmetric patterning of the lateral plate. Given that Gdf1 and Nodal are coexpressed in perinodal cells, the GDF1-Nodal heterodimer likely travels from the node to the lateral plate, where it activates Nodal. This notion is further supported by other observations. First, Nodal possesses two enhancers (ASE and LSE) that confer asymmetric expression in the LPM and both of these enhancers are Nodal responsive (Saijoh et al. 2000, 2005; Vincent et al. 2004). Second, paraxial mesoderm does not express Cripto or Cryptic (Dono et al. 1993; Shen et al. 1997), and so is not able to respond to the Nodal signal. Finally, Cryptic is not required in the node for Nodal expression in the LPM, suggesting that the Nodal signal generated in the node is not relayed between the node and the LPM (Oki et al. 2007).

Interaction with a partner (protein $\mathrm{Y}$ ) is able to enhance the range of a signaling molecule (protein $\mathrm{X}$ ) by at least two different mechanisms (Fig. 7B-D). First, interaction with $\mathrm{Y}$ increases the specific activity of $\mathrm{X}$ without affecting the number of $\mathrm{X}$ molecules that reach a remote target site (Fig. 7C). Alternatively, interaction with $\mathrm{Y}$ may increase the number of $\mathrm{X}$ molecules that reach a remote target site by increasing the diffusion efficiency of $\mathrm{X}$ (Fig. 7D). Our data indicate that interaction with GDF1 markedly increases the specific activity of Nodal, but it remains unclear whether GDF1 also influences the efficiency of Nodal diffusion. To address this latter issue, we introduced an expression vector for Myc epitopetagged Nodal alone or together with an expression vector for Gdf1 into the LPM of mouse embryos and examined the effect of GDF1 on the diffusion of Nodal in the LPM. However, we were unable to detect a substantial effect of GDF1 (data not shown).

Nodal plays essential roles during development, not only in L-R patterning but also in A-P patterning as well as in mesoderm formation and patterning. Gdf1 is expressed ubiquitously in mouse embryos at embryonic days 5.5 and 6.5 (Wall et al. 2000). However, Gdf1 knockout mice exhibit only L-R patterning defects, two explanations of which are possible. First, long-range action of Nodal may not be required for A-P patterning or gastrulation. After establishment of the proximodistal axis in the pregastrulation mouse embryo, for instance, Nodal expression expands from the proximal toward the distal region within the epiblast. This expansion is achieved by an autoregulatory loop mediated by a Nodal-responsive enhancer (Brennan et al. 2001). Given that the epiblast is 
A

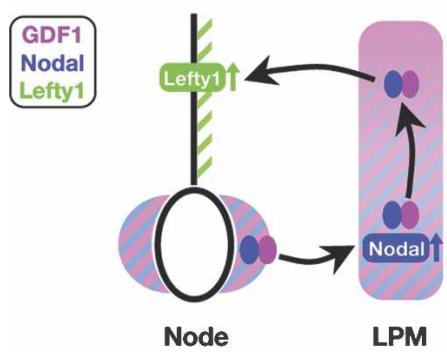

Gdf1 $^{1-}$

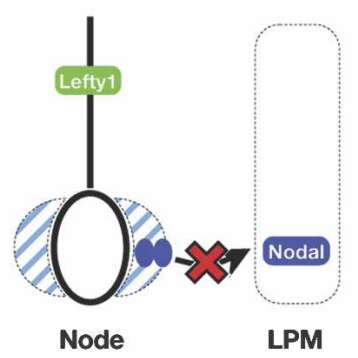

Gdf1 $^{-/-} ;$node-Tg

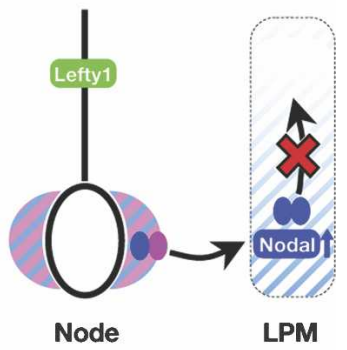

B

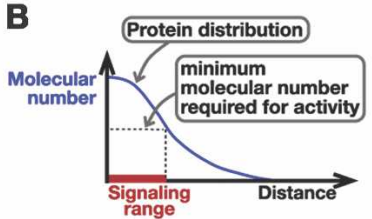

C

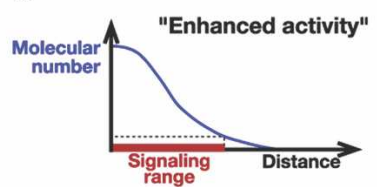

D

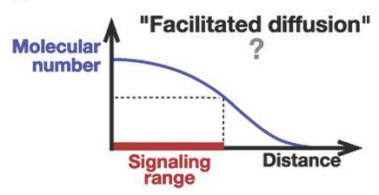

Figure 7. Model for long-range Nodal signaling by a GDF1-Nodal heterodimer during left-side specification in the mouse. $(A$, left panel) In the wild-type embryo, Nodal (blue) and GDF1 (pink) are both expressed in the perinodal region. At the onset of L-R axis formation, an unknown signal generated in the node specifies the left side of the node, and the GDF1-Nodal heterodimer conveys the Nodal signal to the left LPM. The Nodal signal from the node induces Nodal expression in the left LPM, where Gdf1 is already expressed, allowing the local formation of the GDF1-Nodal heterodimer. The GDF1-Nodal heterodimer travels within the LPM along the A-P axis, inducing Nodal expression and resulting in the formation of more heterodimeric complexes. (Center panel) The heterodimer then travels to the midline, where it induces expression of Lefty1 on the left side of the floor plate (green). In the Gdf1 ${ }^{-/-}$ embryo, perinodal cells produce only the Nodal homodimer, which fails to induce Nodal expression in the left LPM. (Right panel) In the $G d f 1^{-/-}$; node-Tg embryo, perinodal cells produce the GDF1-Nodal heterodimer, which induces Nodal expression in the left LPM. The lack of GDF1 in the LPM, however, results in limited Nodal expression in the left LPM and no Lefty1 expression at the midline. $(B-D)$ Models for the mechanism of long-range signaling by morphogens. $(B)$ In the baseline condition, the signaling range of the morphogen is determined by its molecular distribution and its specific activity. $(C)$ An increase in the specific activity of the morphogen reduces the minimum number of molecules required and thus increases the signaling range. $(D)$ Conversely, an increase in the stability or active diffusion of the molecule increases the signaling range without affecting the required activity level.

competent to respond to the Nodal signal, Nodal expression can be extended by sequential amplification over a short range. During gastrulation, Nodal produced in the posterior ectoderm is required to signal to nearby cells in the primitive streak but may not be required to act over a long distance.

Alternatively, the lack of A-P patterning defects and gastrulation defects in $G d f 1$ mutant mice may be due to functional redundancy with a GDF1-related factor. In particular, GDF3 is a member of the TGF- $\beta$ superfamily that is closely related to GDF1 and is expressed in mouse embryonic stem cells and preimplantation embryos. A proportion of $\mathrm{Gdf3^{-/- }}$ mice was recently shown to exhibit A-P patterning defects (Chen et al. 2006). Furthermore, a recent study (Andersson et al. 2006) showed that $\mathrm{Gdf1}^{-/-}$; Nodal ${ }^{+-}$mice exhibit anterior head truncation, indicative of a genetic interaction between $G d f 1$ and Nodal. It is thus possible that GDF3 regulates the activity and signaling range of Nodal during A-P patterning by interacting with Nodal.

\section{Materials and methods}

Generation of transgenic mice

For construction of a transgene (node- $\mathrm{Tg})$ that confers expression of Gdf1 specifically in the perinodal region, two tandem copies of a $0.7-\mathrm{kb}$ DNA fragment containing the NDE of Nodal (Krebs et al. 2003) were linked to the hsp68 promoter, mouse Gdf1 cDNA, and IRES-lacZ. For construction of a transgene (LPM-Tg) that confers Gdf1 expression specifically in the LPM, genomic clones of mouse Cryptic (kindly provided by M. Shen) were analyzed for the presence of an LPM-specific enhancer by the testing of various lac $Z$ reporter constructs in a transgenic assay. The 11-kb upstream region of Cryptic was found to possess such enhancer activity when linked to the hsp 68 promoter and lacZ (Oki et al. 2007). This 11-kb fragment and the hsp68 promoter were therefore linked to Gdf1 cDNA and IRES-IacZ to drive Gdf1 expression in the LPM. The two transgenes were separately microinjected into the pronucleus of fertilized eggs obtained by crossing C57BL/6Cr females with $G d f 1^{+/-}$males (Rankin et al. 2000). Transgenic mice or embryos were identified by PCR analysis of tail or yolk sac DNA, respectively. The specificity and level of transgene expression were monitored by X-gal staining.

\section{Construction of Flag-tagged Nodal and GDF1}

For generation of Flag-tagged GDF1, the Flag epitope tag (DYK DDDDK) was introduced two amino acids downstream from the proteolytic cleavage site of the mouse GDF1 precursor at the DNA level. For generation of Flag-tagged Nodal, a SmaI site was introduced downstream from the DNA sequence encoding the proteolytic cleavage site and an oligonucleotide encoding Flag was then inserted at this restriction site. The inserted sequence contained an extra guanine residue at the $3^{\prime}$ end to prevent a 
frameshift, yielding the amino acid sequence RRQRRHHLPDYKDDDDK-(G)DRS (the proteolytic cleavage site is underlined; additional amino acid residues are in parentheses).

\section{Synthesis and microinjection of synthetic mRNAs and} animal cap assays

The ORFs of genes were cloned into pSP64T (Krieg and Melton 1984), and capped synthetic mRNAs were transcribed with the use of a mMessage mMachine kit (Ambion). For animal cap luciferase assays, each blastomere of four-cell Xenopus embryos was injected at the animal pole. The animal cap was dissected at stage 8.5 , cultured for $3 \mathrm{~h}$, and harvested for assay of luciferase activity with a Luciferase Assay System (Promega). For immunoblot analysis of phospho-Smad2, four animal caps were loaded per lane and probed with rabbit polyclonal antibodies to phospho-Smad2 (Cell Signaling Technology) and a mouse monoclonal antibody to $\alpha$-tubulin (clone DM1A, Sigma). For animal cap lacZ reporter assays, embryos were injected at the 32- or 64-cell stage with reporter or effector mixes together with TRLDx or FLDx (Molecular Probes), respectively, to mark the injected blastomeres (Reilly and Melton 1996). Animal caps were dissected at stage 8.5 , placed in the narrow gap between a slide glass and coverslip, and cultured for $3 \mathrm{~h}$. They were then fixed and stained for $\beta$-galactosidase activity. Stained animal caps were bleached with a solution containing $70 \%$ methanol and $10 \% \mathrm{H}_{2} \mathrm{O}_{2}$ under strong light for several hours for better visualization of staining.

\section{Preparation of conditioned medium from frog oocytes}

Xenopus oocytes were injected with 50 ng of mRNA and then cultured for $3 \mathrm{~d}$ at $20^{\circ} \mathrm{C}$ in modified Barth's solution (MBSH). Conditioned medium was then harvested and used for immunoprecipitation or luciferase assays. For luciferase assays, animal caps injected with the reporter construct were cultured for $3 \mathrm{~h}$ in conditioned medium diluted to $30 \%$ with MBSH containing $0.1 \%$ bovine serum albumin and were then assayed for luciferase activity.

\section{Immunoprecipitation}

Oocyte-conditioned medium $(50 \mu \mathrm{L})$ was mixed with a lysis buffer and subjected to immunoprecipitation with an Anti-Flag M2 Affinity Gel (Sigma) in a total volume of $200 \mu \mathrm{L}$. Immunoprecipitated proteins were resolved by SDS-polyacrylamide gel electrophoresis on a $15 \%$ gel under reducing or nonreducing conditions, and the separated proteins were transferred to a polyvinylidene difluoride filter and subjected to immunoblot analysis with antibodies to GDF1 or to Nodal (generated in rabbits with the mature domain of each protein as the antigen) and with $\mathrm{ECL}^{+}$detection reagents (Amersham).

\section{Gene introduction into mouse embryos}

Full-length cDNAs for mouse GDF1 or Nodal were subcloned into the expression vector pEF-BOS (Mizushima and Nagata 1990). The vector pCX-EGFP (BD Biosciences) was used to mark the site of transfection. For lipofection, plasmids were mixed with LipofectAMINE 2000 (Invitrogen) in $25 \mu \mathrm{L}$ of Opti-MEM (Gibco), as described previously (Yamamoto et al. 2004). Presomitic mouse embryos were dissected, injected with the lipofection solution in the right anterior LPM, and allowed to grow until the five- to six-somitic stage by rotation culture in Dulbecco's modified Eagle's medium supplemented with $75 \%$ rat serum.

\section{Acknowledgments}

We thank Se-Jing Lee (Johns Hopkins University) for Gdf1 mutant mice and GDF1-related reagents, Dan Kessler for zebrafish Squint and $z D V R-1$ cDNAs, Chris Wright for zebrafish Cyclops cDNA, Michael Shen for genomic clones of mouse Cryptic, and Sachiko Ohishi and Hiromi Hashiguchi-Jo for technical assistance. This work was supported by grants from the Ministry of Education, Culture, Sports, Science, and Technology of Japan and by CREST (to H.H.) and the funding from The Eccles Program in Human Molecular Biology and Genetics, University of Utah School of Medicine (to Y.S.). C.T. is a recipient of a fellowship from the Japan Society for the Promotion of Science for Japanese Junior Scientists.

\section{References}

Andersson, O., Reissmann, E., Jornvall, H., and Ibanez, C.F. 2006. Synergestic interaction between Gdf1 and Nodal during anterior axis development. Dev. Biol. 293: 370-381.

Brennan, J., Lu, C.C., Norris, D.P., Rodriguez, T.A., Beddington, R.S., and Robertson, E.J. 2001. Nodal signalling in the epiblast patterns the early mouse embryo. Nature 411: 965-969.

Brennan, J., Norris, D.P., and Robertson, E.J. 2002. Nodal activity in the node governs left-right asymmetry. Genes \& Dev. 16: 2339-2344.

Capdevila, J., Vogan, K.J., Tabin, C.J., and Izpisua Belmonte, J.C. 2000. Mechanisms of left-right determination in vertebrates. Cell 101: 9-21.

Chen, C., Ware, S.M., Sato, A., Houston-Hawkins, D.E., Habas, R., Matzuk, M.M., Shen, M.M., and Brown, C.W. 2006. The Vg1-related protein Gdf3 acts in a Nodal signaling pathway in the pre-gastrulation mouse embryo. Development 133: 319-329.

Cheng, S.K., Olale, F., Bennett, J.T., Brivanlou, A.H., and Schier, A.F. 2003. EGF-CFC proteins are essential coreceptors for the TGF- $\beta$ signals Vg1 and GDF1. Genes \& Dev. 17: 31-36.

Dohrmann, C.E., Kessler, D.S., and Melton, D.A. 1996. Induction of axial mesoderm by zDVR-1, the zebrafish orthologue of Xenopus Vg1. Dev. Biol. 175: 108-117.

Dono, R., Scalera, L., Pacifico, F., Acampora, D., Persico, M.G., and Simeone, A. 1993. The murine cripto gene: Expression during mesoderm induction and early heart morphogenesis. Development 118: 1157-1168.

Hamada, H., Meno, C., Watanabe, D., and Saijoh, Y. 2002. Establishment of vertebrate left-right asymmetry. Nat. Rev. Genet. 3: 103-113.

Kessler, D.S. and Melton, D.A. 1995. Induction of dorsal mesoderm by soluble, mature Vg1 protein. Development 121: 2155-2164.

Krebs, L.T., Iwai, N., Nonaka, S., Welsh, I.C., Lan, Y., Jiang, R., Saijoh, Y., O'Brien, T.P., Hamada, H., and Gridley, T. 2003. Notch signaling regulates left-right asymmetry determination by inducing Nodal expression. Genes \& Dev. 17: 12071212.

Krieg, P.A. and Melton, D.A. 1984. Functional messenger RNAs are produced by SP6 in vitro transcription of cloned cDNAs. Nucleic Acids Res. 12: 7057-7070.

Marques, S., Borges, A.C., Silva, A.C., Freitas, S., Cordenonsi, M., and Belo, J.A. 2004. The activity of the Nodal antagonist Cerl- 2 in the mouse node is required for correct L/R body axis. Genes \& Dev. 18: 2342-2347.

Mizushima, S. and Nagata, S. 1990. pEF-BOS, a powerful mammalian expression vector. Nucleic Acids Res. 18: 5322.

Nonaka, S., Tanaka, Y., Okada, Y., Takeda, S., Harada, A., Kanai, Y., Kido, M., and Hirokawa, N. 1998. Randomization of 
left-right asymmetry due to loss of nodal cilia generating leftward flow of extraembryonic fluid in mice lacking KIF3B motor protein. Cell 95: 829-837.

Oki, S., Hashimoto, R., Otani, H., Shen, M., Saijoh, Y., and Hamada, H. 2007. Sulfated glycosaminoglycan is necessary for Nodal signal transmission from the node to the left lateral plate in the mouse left-right patterning. Development 134: 3893-3904.

Pearce, J.J., Penny, G., and Rossant, J. 1999. A mouse cerberus/ Dan-related gene family. Dev. Biol. 209: 98-110.

Rankin, C.T., Bunton, T., Lawler, A.M., and Lee, S.J. 2000. Regulation of left-right patterning in mice by growth/differentiation factor-1. Nat. Genet. 24: 262-265.

Reilly, K.M. and Melton, D.A. 1996. Short-range signaling by candidate morphogens of the TGFb family and evidence for a relay mechanism of induction. Cell 86: 743-754.

Saijoh, Y., Adachi, H., Sakuma, R., Yeo, C.Y., Yashiro, K., Watanabe, M., Hashiguchi, H., Mochida, K., Ohishi, S., Kawabata, M., et al. 2000. Left-right asymmetric expression of lefty 2 and nodal is induced by a signaling pathway that includes the transcription factor FAST2. Mol. Cell 5: 35-47.

Saijoh, Y., Oki, S., Ohishi, S., and Hamada, H. 2003. Left-right patterning of the mouse lateral plate requires nodal produced in the node. Dev. Biol. 256: 160-172.

Saijoh, Y., Oki, S., Tanaka, C., Nakamura, T., Adachi, H., Yan, Y.T., Shen, M.M., and Hamada, H. 2005. Two nodal-responsive enhancers control left-right asymmetric expression of Nodal. Dev. Dyn. 232: 1031-1036.

Sakuma, R., Ohnishi, Y., Meno, C., Fujii, H., Juan, H., Takeuchi, J., Ogura, T., Li, E., Miyazono, K., and Hamada, H. 2002 Inhibition of Nodal signalling by Lefty mediated through interaction with common receptors and efficient diffusion. Genes Cells 7: 401-412.

Shen, M.M., Wang, H., and Leder, P. 1997. A differential display strategy identifies Cryptic, a novel EGF-related gene expressed in the axial and lateral mesoderm during mouse gastrulation. Development 124: 429-442.

Thomsen, G.H. and Melton, D.A. 1993. Processed Vg1 protein is an axial mesoderm inducer in Xenopus. Cell 74: 433-441.

Vincent, S.D., Norris, D.P., Le Good, J.A., Constam, D.B., and Robertson, E.J. 2004. Asymmetric Nodal expression in the mouse is governed by the combinatorial activities of two distinct regulatory elements. Mech. Dev. 121: 1403-1415.

Wall, N.A., Craig, E.J., Labosky, P.A., and Kessler, D.S. 2000. Mesendoderm induction and reversal of left-right pattern by mouse Gdf1, a Vg1-related gene. Dev. Biol. 227: 495-509.

Yamamoto, M., Mine, N., Mochida, K., Sakai, Y., Saijoh, Y., Meno, C., and Hamada, H. 2003. Nodal signaling induces the midline barrier by activating Nodal expression in the lateral plate. Development 130: 1795-1804.

Yamamoto, M., Saijoh, Y., Perea-Gomez, A., Shawlot, W., Behringer, R.R., Ang, S.L., Hamada, H., and Meno, C. 2004. Nodal antagonists regulate formation of the anteroposterior axis of the mouse embryo. Nature 428: 387-392. 


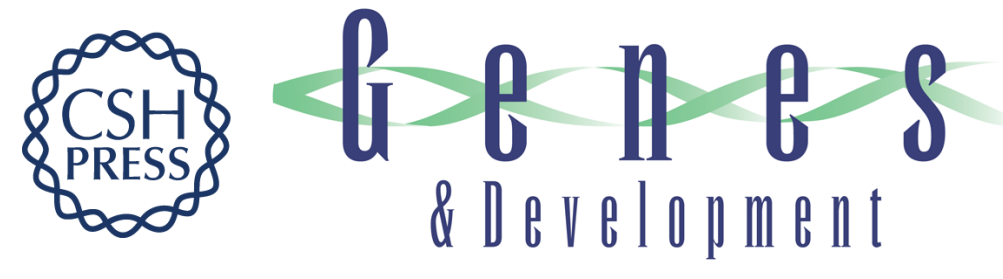

\section{Long-range action of Nodal requires interaction with GDF1}

Chinatsu Tanaka, Rui Sakuma, Tetsuya Nakamura, et al.

Genes Dev. 2007, 21:

Access the most recent version at doi:10.1101/gad.1623907

Supplemental
Material http://genesdev.cshlp.org/content/suppl/2007/11/28/21.24.3272.DC1

References This article cites 29 articles, 10 of which can be accessed free at: http://genesdev.cshlp.org/content/21/24/3272.full.html\#ref-list-1

License

Email Alerting Receive free email alerts when new articles cite this article - sign up in the box at the top Service right corner of the article or click here.

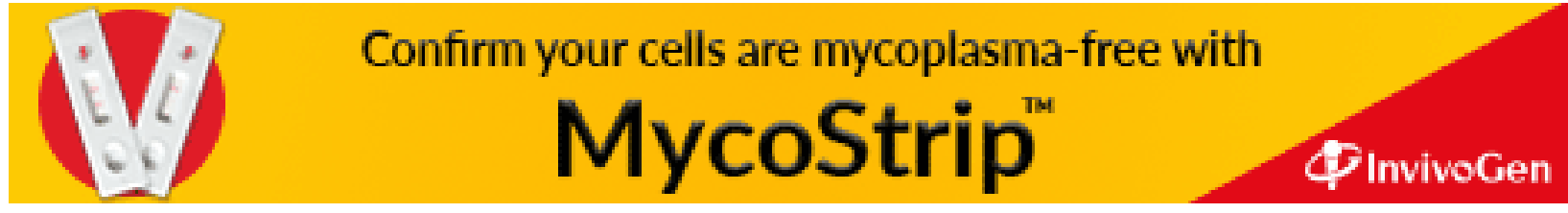

